\title{
Self-Inflicted Multiple Organs Injury with Nail Gun
}

\author{
Xiaorong Fang, BN, ${ }^{1}$ Changjiang $\mathrm{Yu}, \mathrm{MD},{ }^{2} \mathrm{Fan} \mathrm{He}, \mathrm{MD}^{2}$ \\ ${ }^{1}$ Department of Thoracic Surgery, Sir Run Run Shaw Hospital, School of Medicine, Zhejiang University, Hangzhou, China; \\ ${ }^{2}$ Department of Cardiovascular Surgery, Sir Run Run Shaw Hospital, School of Medicine, Zhejiang University, Hangzhou, China
}

\section{ABSTRACT}

We report the case of a patient with injuries to multiple organs as a result of attempted suicide with a nail gun. The patient shot 12 nails into his chest, causing damage to multiple organs, including the heart, lungs, and stomach. With timely emergency surgery, we successfully removed all the nails, and the patient was discharged from hospital two weeks after surgery.

\section{INTRODUCTION}

Nail guns are commonly used power tools for industrial and domestic purposes. In medical literature, nail guns mostly have been described as causing orthopedic injuries of the non-dominant arm. However, life threatening cardiac injuries also have been reported, especially self-inflicted cases [Ho 2017]. Here, we report the case of a 23-year-old man who attempted to commit suicide by shooting more than 10 nails into his chest with a nail gun.

\section{CASE REPORT}

A 23-year-old male patient was admitted to the emergency department of our hospital by his father, due to chest pain and because he was pale. Physical examination on admission showed stable vital signs without him losing consciousness (Glasgow Coma Scale: E4V5M6) and limb weakness (muscle strength with grade 4). Several scars and local eminence could be seen on his left chest wall. (Figure 1) Respiratory sounds of both lungs could be heard with wet rales on auscultation. The results of laboratory examination showed that C-reactive protein was $319.9 \mathrm{mg} / \mathrm{L}$, white blood cell count was $18.9 \times 10^{\wedge} 9 / \mathrm{L}, \mathrm{N}$-terminal pro brain natriuretic peptide was $697 \mathrm{pg} / \mathrm{ml}$, and troponin I was $0.87 \mathrm{ng} / \mathrm{ml}$. Electrocardiogram results showed short PR interval and extensive ST segment elevation. Urgent CT examination revealed exudation in the left lung and bilateral pleural effusion, especially

Received August 5, 2021; accepted September 15, 2021.

Correspondence: Fan He, MD, East Qingchun Road 3, Hangzhou, Zhejiang Province, China (e-mail: hefan8917@zju.edu.cn). at the left. High density shadows in the left anterior chest wall, heart, left lung, and stomach were considered foreign bodies. (Figure 2) Massive pericardial effusion also was presented (Figure 2B, 2C). Bedside echocardiography also indicated a large pericardial effusion, of which the widest part of the apex was about $12 \mathrm{~mm}$. We performed emergency surgery on the patient through cardiopulmonary bypass. A total of 12 nails, about $5 \mathrm{~cm}$ in length, were found (Figure 3A). (Figure 3) Of these 12 nails, six penetrated the chest wall and the left ventricular wall, three nails penetrated the middle and lower lobe of the left lung, two nails were located in the chest wall, and the other nail was found to be located near the gastric fundus on the anterior wall of the upper gastric body through gastroscopy, with one side free and the other side trapped in the gastric wall (Figure 3B).

Emergency surgery was performed with a median sternal incision assisted by cardiopulmonary bypass. Eleven nails were removed during the operation, and the ruptured left ventricle and middle and lower lobe of the left lung were repaired. The nail in the stomach was removed endoscopically, and the gastrointestinal decompression tube was indwelling. The operation was successful, and the patient successfully was discharged two weeks after the operation.

\section{DISCUSSION}

The first nail gun injury was reported in 1959, the year in which the device was introduced into the construction industry [Hoffman 1997]. With the wide application of nail guns in our industry and life, the incidence of nail gun injuries also is steadily increasing. Most of the injuries occur in the upper limbs, primarily in the fingers and hands. Many injuries are related to misuse of nail guns and are not accidental injuries [Litvack 2006; Bonatti 2020]. However, a systematic review found that more than 65 percent of nail gun injuries were related to suicide attempts [Woodall 2012]. The mortality rate of penetrating cardiac injury varies depending on the object of penetration. Nail gun injuries penetrating the heart have a mortality rate of up to $25 \%$. Mortality rates of stabbing injuries of the heart range from $22 \%$ to $62 \%$. Gunshot injuries have a higher mortality rate from $60 \%$ to $95 \%$ [Ho 2017]. The mortality rate may be higher for nail injuries involving multiple organs, including the heart, lungs, and stomach. Clinical manifestations of nail gun injuries penetrating the heart range from asymptomatic to cardiac hemorrhage and cardiac tamponade [Jodati 2011]. Thoracoabdominal penetrating injuries may also 


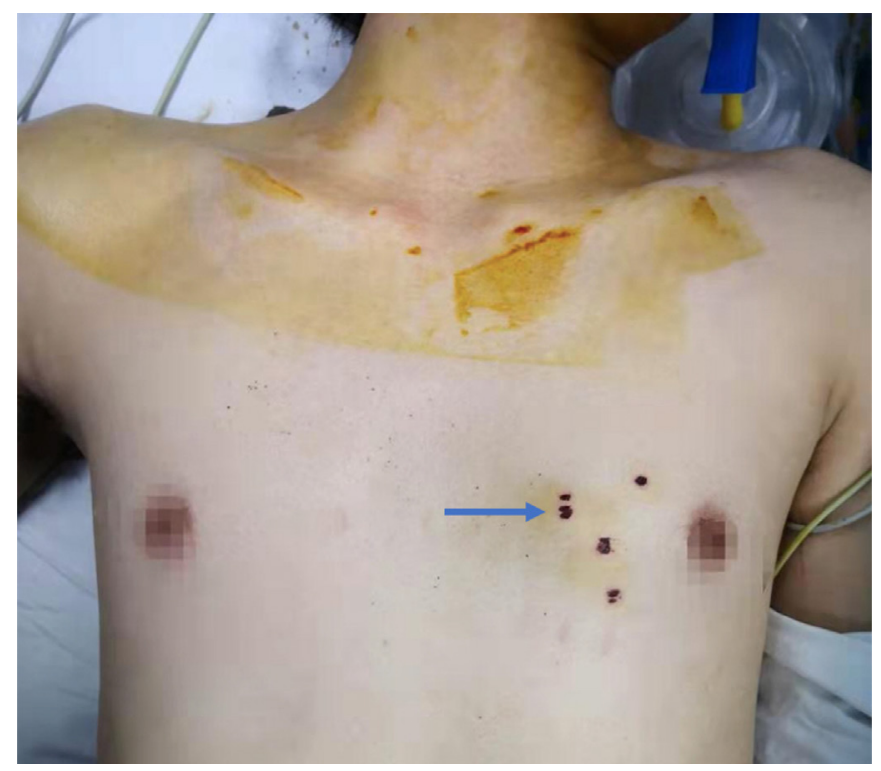

Figure 1. Several scars and local eminence could be seen on the patient's left chest wall.

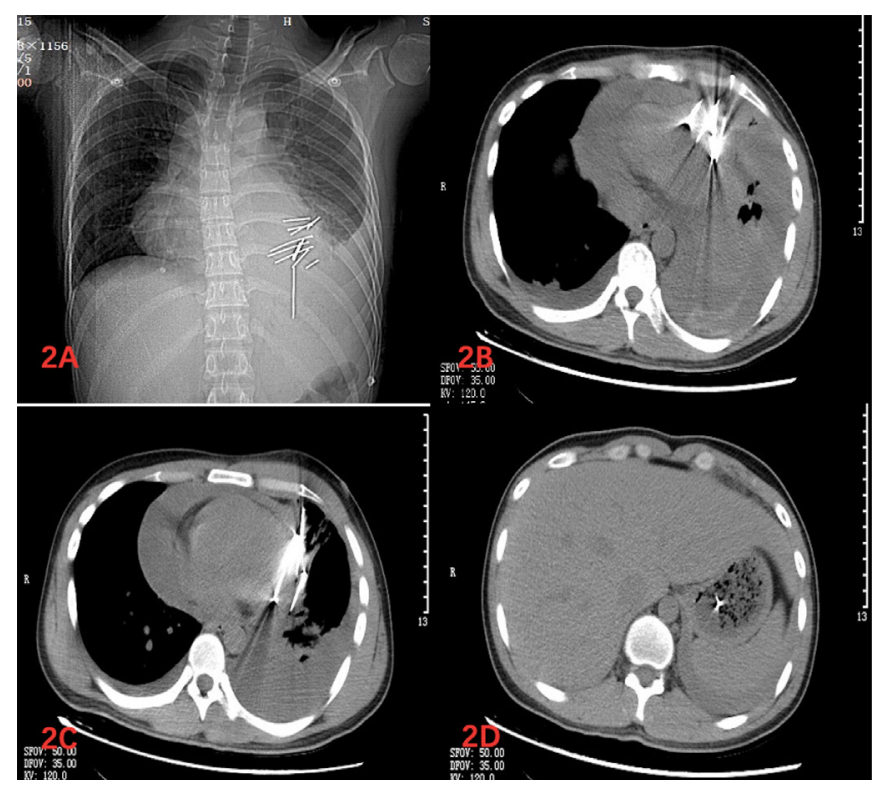

Figure 2. A-D) CT examination showed that nail shadows were found in the left anterior chest wall, heart, left lung, and stomach. B, C) Massive pericardial effusion was found.

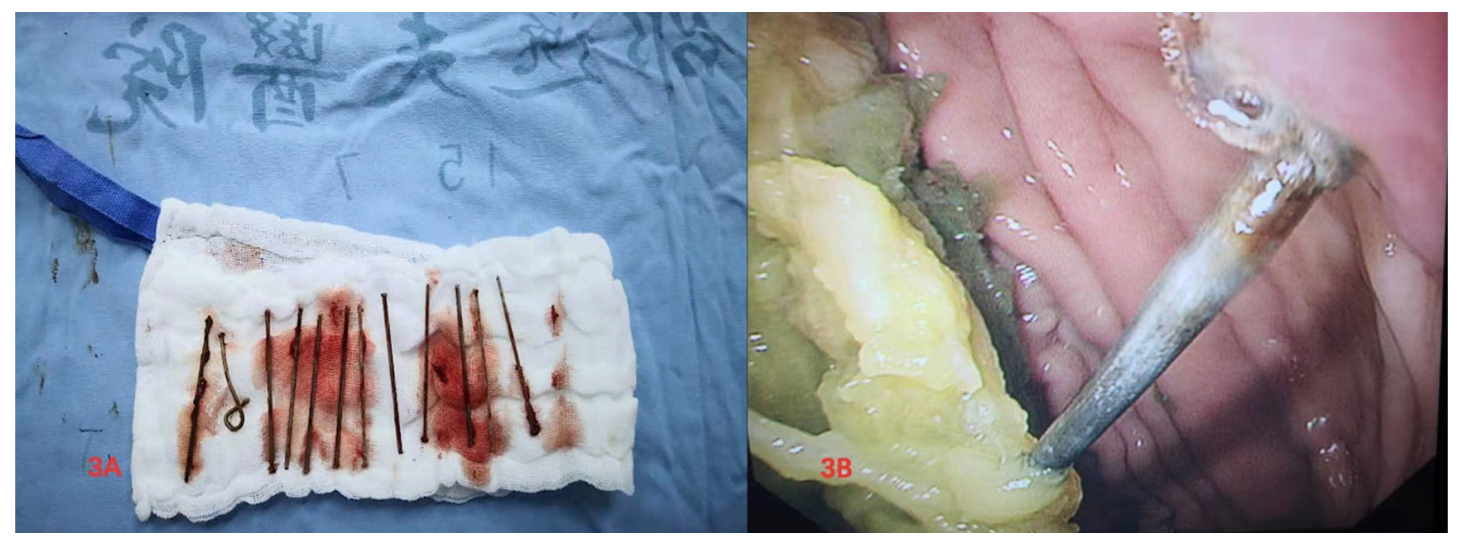

Figure 3. Twelve nails, about $5 \mathrm{~cm}$ in length, were removed during the operation. A) Gastroscopy indicated that one nail was located near the gastric fundus on the anterior wall of the upper gastric body, with one side free and the other side trapped in the gastric wall (B).

present symptoms, such as hemoptysis, hemothorax, shortness of breath and hematemesis, which may be due to the wide range of muzzle energy of the nail gun, lack of stability, and low accuracy.

Surgical treatment is the accepted treatment option for patients with nail injuries. For patients with stable hemodynamics, conventional chest radiography, CT and echocardiography can help diagnose and determine surgical treatment. For patients with unstable hemodynamics, emergency exploratory thoracotomy should be performed. Intraoperative transesophageal echocardiography has been reported to help guide surgical treatment [Madani 2012; Tuladhar 2009]. Yamamoto et al. believe that cardiopulmonary bypass is a safe alternative for maintaining hemodynamic stability, identifying the exact site of damaged vessels, and safely repairing injuries during surgery [Yamamoto 2020]. For our patient with multiple organ nail gun injuries to the heart, lung and stomach, we used cardiopulmonary bypass to remove the nails and repair the heart and lung and combined with gastroscopy to remove the nails from the stomach and postoperative gastrointestinal decompression therapy. The successful postoperative recovery of the patient confirmed that our treatment was effective.

\section{CONCLUSION}

Multiple organ injuries due to gun nails caused by attempted suicide are relatively rare. CT and echocardiography are effective in diagnosis and treatment. Operation under 
cardiopulmonary bypass is an effective treatment for maintaining hemodynamic stability, identifying the exact site of damaged vessels, and safely repairing injuries during surgery.

\section{REFERENCES}

Bonatti H. 2020. He nailed it: Nail gun injury to the transverse colon. Laparosc Endosc Robot Surg. 3(3):85-89.

Ho S, Liu B, Feranec N. 2017. Self-inflicted Cardiac Injury with Nail Gun Without Hemodynamic Compromise: A Case Report. Cureus, 9(1), e971.

Hoffman DR, Jebson PJ, Steyers CM. 1997. Nail gun injuries of the hand. Am. Fam. Physician. 56: 1643-6.

Jodati A, Safaei N, Toufan M, Kazemi B. 2011. A unique nail gun injury to the heart with a delayed presentation. Interact. Cardiovasc. Thorac. Surg. 13: 363-5.

Litvack ZN, Hunt MA, Weinstein JS, et al. 2006. Self-Inflicted nail-gun injury with 12 cranial penetrations and associated cerebral trauma. Case report and review of the literature.J Neurosurg. 104:828-34.

Madani M, Drissi M, Ajaja MR, et al. 2012. Nail gun may cause heart injury: a young adult's misadventure. Int Emerg Nurs. 20:98-101.

Tuladhar S, Eltayeb A, Lakshmanan S, Yiu P. 2009. Delayed presentation of right and left ventricle perforation due to suicidal nail gun injury. Ann Card Anaesth. 12:136-9.

Woodall MN, Alleyne CH. 2012. Nail-Gun head trauma: a comprehensive review of the literature. J Trauma Acute Care Surg. 73:993-6.

Yamamoto A, Umeda Y, Kamei Y, et al. 2020. Penetrating nail-gun injury of the thoracic descending aorta. [J]. Acute Med Surg. 7: e530. 